\title{
Enhancing Micro-ATMs and POS Terminals Authentication System Using AdvancedBiometric Techniques
}

\author{
*Priya Tawde ${ }^{1}$, Dr. G. Prasanna Lakshmi ${ }^{2}$ \\ ${ }^{1}$ Research Scholar,Faculty of Science,Pacific University, Udaipur \\ ${ }^{2}$ Guide (WOS-A) Andhra University
}

\begin{abstract}
Most of the transactions at the Point of Sale (POS) terminals are carried out by payments through Credit or Debit cards.Many leading banks have started the door step banking service with the help of microATM device.The current authentication system uses fingerprint authentication or PIN based authentication method.Biometric features are unique for every individual and hence can be widely used in fusion for enhancing the security system for micro-ATMS and POS terminals.
\end{abstract}

Keywords: Biometric authentication, Micro-ATM Security, Point of sale terminal biometric authentication.

\section{Introduction}

In today's age of ecommerce and digital banking most of the transactions at the Point of Sale (POS) terminals are carried out by payments through Credit or Debit cards. After the demonetisation of currency notes in November 2016 there is huge increase in the card payments instead of cash transactions at any POS terminal irrespective of the amount whether it is small or huge amount.The current system of authentication at any Point of Sale terminal is based on Personal Identification Number (PIN) which the user inputs on the handheld card swiping machine connected to the billing system. At the time of inputting the 4 digit PIN number the people standing around the billing system can easily have a glance of what the user is entering in machine and thus making the system less secure and uncomfortable for the user. Also the advancement of technology has helped the fraudsters to create skimming machines which can copy the entire data of customer from the card and this data can be used to create duplicate card and the pin is acquired by observation when the card holder inputs the PIN or by a hidden camera which captures the input. Biometric authentication comes as a remedy for such types of frauds.

Many leading banks have started the door step banking serviceswith the help of micro-ATM device which is a handheld palm top device which can provide services like a traditional ATM machine.The account holders can visit the service centre of the banks available at rural areas for cash withdrawals or third party transfers with the help of debit card. These centres are equipped with micro-ATM devices which are battery operated and are GPRS enabled. The micro-ATM device has a finger print sensor attached which can capture finger print input. The account holder swipes his debit/credit card in the machine and a One Time Password (OTP) is sent to the registered mobile number of account holder. If the finger print of account holder is not recognised due to hard skin or marks of cut on the skin then this OTP is used to authenticate the user and complete the transaction. This makes the system less secure as biometric authentication is not mandatory and an alternative OTP is used.

\section{Related Work}

S. Koteswari, P. John Paul, AgshareDheeraj and Rajesh Kone (2016) have analysed the existing problems of using Personal Identification Number (PIN) based authentication. To enhance the security of ATM system they have suggested a high level model which uses combination of security protocols as PIN and biometric fingerprint along with iris strategy. They have proposed a multimodal mechanism as a biometric measure to enhance the security features of the ATM for effective banking transaction for Indian banking system.Mr. MuleSandip S., Mr. H. B. Mali (2015) have explained various techniques of biometric authentication using human's physiological and behavioural characteristics. Biometric authentication can be done using fingerprint, eye patterns (IRIS recognition), hand geometric, facial expression, voice recognition, and signature analysis etc. They have emphasized on the future need and scope of Palm print recognition system for authentication.K.Senthil Kumar, Dr.S.Vijayaragavan(2014) have focused on the issues of fixing ATM card scanner in ATM machine. Many incidences have reported where the ATM machines have been fixed with ATM skimming machines to capture the encrypted data of user ATM cards and then this data is further used to create duplicate cards. These duplicate cards are used by fraudsters to carry out fraud transactions. They have proposed a system with combination of Biometric, Face recognition and retina (IRIS) to authenticate user in ATM machine. Their proposed system uses three step authentication. Their proposed system also helps in eliminating the hidden cost of card management. 
Mr.MahesPatil, Mr. Sachin P Wanere, Mr.RupeshMaighame and Mr.Akshay Tiwari (2012) have elaborated the use of ATM transaction with biometric authentication using Fingerprint. They have thrown light on the issues such as password and PIN can be stolen by observers who are around the transaction points. They have chosen biometric authentication such as fingerprint as this data is owned by its user and is non-transferable and unique to each individual. They have focused on the advantage of fingerprint due its stability and reliability.V.Padmapriya, S.Prakasam(2013) have made a comparison of various ways of using finger print as biometric authentication parameter. They have compared the minutia score matching method and user authentication based on elliptical curve. They have proposed an enhanced ATM security using fingerprint and GSM techniques. Their proposed system authenticates the user by biometric method and if it fails then an alternate method of GSM technology is used to generate One Time Password via registered mobile number of account holder.G. ManoharBabu, R. Anil Kumar (2015) have explained how users have to carry multiple ATM cards for various banks. If a user has multiple accounts in various banks he has to carry those cards to the ATM. Also the user is levied transaction charges for using different cards in a single ATM machine. They have proposed a system which allows access to multiple bank accounts of a user with single smart card which can be used for ATM transactions. This will reduce the problem of carrying multiple cards and memorising PIN for those cards. They have proposed system with Embedded Smart Card ATM by using ARM7 Based RFID \& GSM Technology for Multiple Banking System Accessing.

MythreyaSeetharama, Volker Paelke, and Carsten Rocker (2015) have raised concern over the PIN based authentication in which the user has to input PIN manually. They have proposed a system which will catch the eye movement of the user and input the PIN as per eye movement over the screen showing keypad display. They have used the eye tracking system for recording the input. This will prevent the observers from knowing the PIN while the user inputs PIN using eye tracking technology.K. Nirosha, T. Manivannan (2016)have proposed a framework to enhance the security of ATM clients by making use of Finger Print Recognition System module. They are suggesting a framework by using ARM 7 LPC2148 controller to control the entire framework and Finger Print Recognition System module to uniquely mark check and approve ATM account administrations. Rushikesh Kulkarni, MuzammilMadki and TejasMapari (2016) have stated that the current ATM system which uses PIN based authentication is having drawbacks of skimming, Lebanese loops, card theft etc. They have proposed a card less ATM system where the user will input his finger print on the system instead of ATM card and then authenticate his account using PIN. For this they propose that the banks should maintain database with users email id, and finger print scan images in the bank servers database. The customer will be provided with account number and PIN number by the bank. There won't be any need of ATM card so this will make the system more secure over the current existing ATM system which uses card and PIN authentication.

\section{Biometric Techniques}

Biometric Features refers to the unique physiological features which an individual has such as iris pattern, fingerprints, facial structure, voice modality and tonality. These physiological features are distinct for each and every individual andcannot be copied easily thus making them highly recommendable to be used as authentication tool for many digital devices and softwares. Different types of biometric measures are as follows: Eyes - Iris Recognition: It is visual biometric feature to identify an individual by his iris pattern.

Eyes -Retina Recognition: It is visual biometric feature which makes use of patterns of veins in the back of the eye to accomplish recognition.

Face Recognition: It is visual biometric feature which analysis the facial features or patterns for recognising an individual.

Fingerprint Recognition: It is visual biometric feature which uses the ridges and valleys (minutiae) that can be traced on the surface tips of a persons fingers for identification.

Also there are other ways such as palm vein recognition, Hand geometry for identification of an individual using biometric features.

\section{Proposed Work}

To enhance the security of account holder and make transactions at the micro-ATMs and POS terminals highly secure with advanced biometric authentication system we propose to make fingerprint recognition mandatory along with PIN based authentication. The banks can acquire the fingerprint data of users while opening account of the user which can be used to match with the fingerprint input given at the microATM or POS terminal during transaction for verification. We also propose that banks can acquire the finger print data of nominee of the account holder additionally to provide facility of usage of debit card or credit card by the nominee where the primary account holder cannot be present due to some physical limitations or any other reasons. In such case when the nominee authenticates the transaction with his finger print a One Time Password will be sent to the registered mobile number of primary account holder so that the account holder is 
kept well informed and the nominee does not misuse the authority which is given to him for emergency cases. This One Time Password will be input at the terminal and then only the transaction is authenticated and completed.

Working of the proposed system:

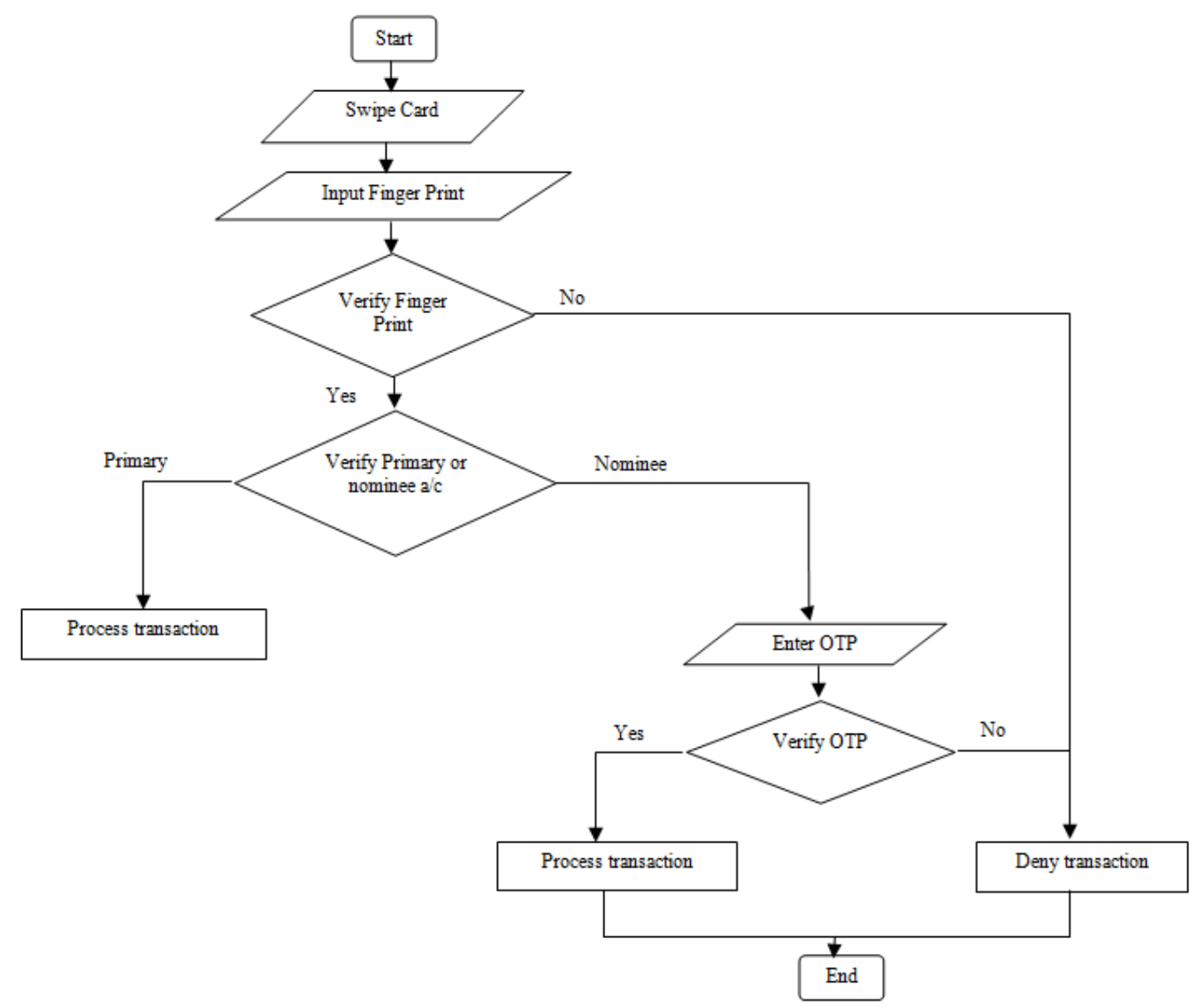

Fig.1. Proposed biometric authentication system at POS swipe machine.

The advantages of the proposed system over traditional system are as follows:

- As the finger print features cannot be copied or stolen so that the issue of misusing the card are ruled out and the transaction becomes more secure and safe.

- The biometric authentication is very easy for account holder as there is no need to remember or memorise the passwords or PIN numbers.

- The hardware required for fast computing of the finger print scanning makes the feature more popular than the other biometric features.

- The availability of low cost finger print capturing devices makes it more convenient and easy to install along with the swiping machines.

\section{Conclusion}

Biometric features are unique for every individual and hence can be widely used in fusion for enhancing the security system for micro-ATMS and POS terminals. In the proposed system biometric authentication is made mandatory for verification of the account holder at the Point of Sale terminal or while using micro-ATM device for banking services. The compulsory biometric authentication will help in making the security of the account holder stronger and will eliminate the frauds up to large extent. Also if the account holder makes use of registering the nominee for his account then the biometric authentication can be supported by sending One Time Password on registered mobile number of the primary account holder to make the transactions transparent and in benefit of the account holder. 


\section{References}

[1]. S.Koteswari, Dr.P.John Paul: “A Survey: Fusion of Fingerprint and Iris for ATM services”, International Research Journal of Engineering and Technology (IRJET), Volume: 04 Issue: 01 | Jan -2017, e-ISSN: 2395 -0056

[2]. AlirezahFarhang, Hasan Rashidi: “ATM Security based on Fingerprint Biometric and SVM", International Journal of Computer \& Information Technologies (IJOCIT), February 2016, ISSN = 2345-3877

[3]. S.PadmaPriya: "Biometrics and Fingerprint Payment Technology", International Journal of Advanced Research in Computer Science \& Technology (IJARCST 2017), Vol. 5, Issue 1 (Jan. - Mar. 2017) ISSN : 2347 - 9817

[4]. AnshuPremchand, Anurag Choudhry: "Future of Payments- ePayments", International Journal of Emerging Technology and Advanced Engineering,ISSN 2250-2459, ISO 9001:2008 Certified Journal, Volume 5, Issue 1, January 2015

[5]. Mr. Mahesh A. Patil, Mr. Sachin P. Wanere, Mr.RupeshP.Maighane, Mr.AashayR.Tiwari : "ATM Transaction Using Biometric Fingerprint Technology",International Journal of Electronics, Communication \& Soft Computing Science and Engineering, ISSN: 2277-9477, Volume 2, Issue 6, 2012

[6]. GazalBetab, Ranjeet Kaur Sandhu: "Fingerprints in Automated Teller Machine-A Survey",International Journal of Engineering and Advanced Technology (IJEAT), ISSN: 2249 - 8958, Volume-3, Issue-4, April 2014

[7]. V.Padmapriya, S.Prakasam : "Enhancing ATM Security using Fingerprint and GSM Technology", International Journal of Computer Applications (0975 - 8887), Volume 80 - No 16, October 2013

[8]. K.Senthil Kumar, Dr.S.Vijayaragavan: "New Secured Architecture for Authentication in Banking Application",International Journal of Innovative Research in Science, Engineering and Technology,ISSN Online (2319-8753)Print(2347-6710), Vol. 3, Issue 2, February 2014

[9]. Jaswinder Singh, Jaswinder Kaur: "Proposed Security System To Embed Fingerprinting and Voice Recognition For ATMs", International Journal of Advanced Research in Computer Science and Software Engineering, Volume 5, Issue 5,May2015 ISSN: $2277128 \mathrm{X}$

[10]. MythreyaSeetharama , Volker Paelke, and Carsten Rocker : "SafetyPIN: Secure PIN EntryThrough Eye Tracking", Springer International Publishing Switzerland 2015

[11]. Rushikesh Kulkarni, MuzammilMadki and TejasMapari : “CARD-LESS ATM SYSTEM”, International Education and Research Journal, E-ISSN No : 2454-9916, Volume : 2, Issue : 4, April 2016

[12]. SiamakSolat : "Security of Electronic Payment Systems: A Comprehensive Survey", Sorbonne Universites, UPMC University of Paris VI, French National Centre for Scientific Research CNRS, Computer Laboratory January 2017, pp.29 -29

[13]. OmprakashKaiwartya, Mukesh Prasad, Shiv Prakash, DurgeshSamadhiya, Abdul Hanan Abdullah, Syed OthmawiAbd Rahman: "An Investigation on Biometric Internet Security”, International Journal of Network Security, Vol.19, No.2, PP.167-176, Mar. 2017

[14]. Sakshi Singh, SimarnjeetKaur: "Enhancing ATM Security Using Fusion of Biometric Techniques", IITM Journal of Management and IT, National Conference on Emerging Trends in Information Technology Cyber Security: Contemporary Threats and Solutions, Volume 7 Issue 1 January-June, 2016

[15]. Vanesa Daza, Roberto Di Pietro, Flavio Lombardi, and Matteo Signorini: "FRoDO: Fraud Resilient Device for Off-line micropayments", IEEE Transactions on Dependable and Secure Computing, DOI 10.1109/TDSC.2015.2432813

[16]. G. ManoharBabu, R. Anil Kumar : "Multiple Banking System Accessing with Embedded Smart Card ATM by using ARM7 Based RFID \& GSM Technology”,International Journal of Advanced Technology and Innovative Research,vol.07, Issue.08, July 2015

[17]. Ram Garg, NK Garg: “Developing a Secured Biometric Payments Model Using Tokenization”, White Paper, www.rsystems.com

[18]. S.Kannan and V. Seenivasagam: "Enhanced Biometric Authentication Using Multi Feature Signature Resemblance and Multi View Edge Sectional Similarity", Asian Journal of Information Technology, 2016, IISN: 1682-3915

[19]. Robert Stokes, Angelica Willis, Kelvin Bryant, Zanetta Tyler, and Anthony Dobson : "Comparison of Biometric Authentication Software Techniques: GEFE vs. Angle Based Metrics", MAICS 2016

[20]. Akhilesh Singh, Sweta Singh,Rakesh Kumar: "Secure swipe machine with help of biometric security", International Conference on Electrical, Electronics and Optimization Techniques (ICEEOT) - 2016

\section{Biography:}

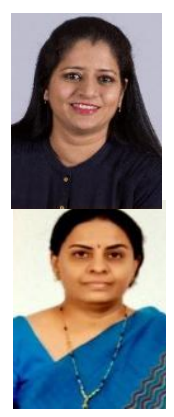

Mrs.Priya Tawde has completed her Master's Degree in Computer Science and Applications from University of Mumbai. Currently she is pursuing her Ph.D. from Pacific University, Udaipur.

Dr. G.Prasanna Lakshmi, Guide. Women Scientist (WOS-A) Andhra University.

IOSR Journal of Computer Engineering (IOSR-JCE) is UGC approved Journal with Sl. No. 5019, Journal no. 49102.

Priya Tawde. "Enhancing Micro-ATMs and POS Terminals Authentication System Using AdvancedBiometric Techniques." IOSR Journal of Computer Engineering (IOSR-JCE) 19.4 (2017): 74-77. 